\title{
Inversion of STEM EELS Data to Obtain Site Occupancy and Near Edge Structure.
}

\author{
Mark P. Oxley ${ }^{1,2}$, Melissa J. Neish ${ }^{3}$, Leslie J. Allen ${ }^{3}$ and Matthew F. Chisholm ${ }^{2}$ \\ 1. Department of Physics and Astronomy, Vanderbilt University, Nashville USA. \\ 2. Materials Science and Technology Division, Oak Ridge National Laboratory, Oak Ridge, USA. \\ 3. School of Physics, University of Melbourne, Melbourne, Australia
}

Aberration correction has led to routine scanning transmission electron microscope (STEM) images based on electron energy loss spectroscopy (EELS) with atomic resolution [1]. Such images are often obtained from very thin specimens, but not all specimens can be prepared in a way that leads to simple visual interpretation. Even for thin specimens, the interpretation of data is complicated by probe spreading due to both elastic and inelastic scattering processes [2]. Specimens prepared by ion milling are usually of the order of 40-60 nm thick and can be particularly hard to obtain clearly resolved spectrum images, even though the underlying structure is clearly seen in annular dark field (ADF) images.

MnFePSi compounds exhibit a giant magnetocaloric effect, which makes them prime candidates for solid state refrigeration. These compounds are based on the hexagonal $\mathrm{Fe}_{2} \mathrm{P}$ structure where the Fe atoms occupy two distinct sites, with tetragonally coordinated $3 f$ sites and pyramidally coordinated $3 g$ sites, which we shall label Fe1 and Fe 2 respectively. The addition of $\mathrm{Mn}$ and $\mathrm{Si}$ at varying levels can be used to improve the properties of these compounds. Studies using density functional theory and neutron scattering predict the Mn preferentially occupies the $\mathrm{Fe} 2$ site $[3,4]$. In this work we will examine $\mathrm{Mn}_{0.43} \mathrm{Fe}_{1.57} \mathrm{P}_{0.73} \mathrm{Si}_{0.27}$ using real space STEM EELS measurements to determine the position of the Mn atoms and the variation of the $\mathrm{Fe} \mathrm{L}_{23}$ ratio between the Fe1 and Fe 2 sites. To overcome the effects of both multiple elastic scattering and thermal diffuse scattering of the STEM probe we apply a recently developed inversion technique to obtain the underlying inelastic scattering potential from STEM EELS data obtained using an aberration corrected Nion UltraSTEM operating at $200 \mathrm{kV}$ [5].

In Fig. 1 we show simultaneously acquired ADF (a) and EEL spectrum images based on the Fe L shell (b) and Mn L shell (c). The hexagonal structure is clearly seen in (a) and the structure is overlaid on an ADF simulation seen in (d). A probe forming aperture of $30 \mathrm{mrad}$. and an EELS collection semi-angle of $36 \mathrm{mrad}$. have been used. The specimen thickness was determined to be $65 \mathrm{~nm}$ from low-loss EELS data. While the positions of the Fe columns may be inferred from Fig. 1(b), the Mn L spectrum image in (c) does not provide any visual identification of the Mn sites. After the inversion procedure, the Fe atomic columns can be clearly seen in the recovered scattering potential shown in Fig. 1(e) with the Fe1 columns identified by the blue circles and the Fe 2 columns by the red circles. Similarly the Mn L scattering potential shown in 1(f) clearly shows the preferential occupancy of the Fe2 sites by the Mn impurities.

Not only are we able to determine the location of the Mn impurities, but the near edge structure of the different Fe sites can also distinguished. In Fig. 2(a) the averaged Fe L shell spectrum obtained directly from the experimental data is shown for both the Fe1 (blue lines) and Fe 2 columns (red lines). There is a slight reduction in the Fe1 L3 peak compared to that of the Fe 2 spectrum. This difference becomes far more distinct in the averaged spectra obtained from the Fe L-shell potentials obtained by inversion shown in Fig 2(b). 
This technique provides real space visualization of site occupancy and unlike diffraction based techniques, promises local site identification rather than averaged site occupancies.

\section{References:}

[1] M Bosman et al, Phys. Rev. Lett. 99 (2007) 086102.

[2] B D Forbes et al., Phys. Rev. B 86 (2012), 024108.

[3] N H Dung et al., Adv. Energy Mater. 1 (2011), p. 1215.

[4] Z Q Ou et al., Magn, Magn. Mater. 340 (2013), p. 80.

[5] N. Lugg et al., Appl. Phys. Lett. 101 (2012), 183112.

[6] This research was supported under the Discovery Projects funding scheme of the Australian Research Council (Project No. DP110102228). This work was sponsored by US Department of Energy, Office of Science, Basic Energy Sciences, Materials Sciences and Engineering Division and supported in part by DOE Grant No. DE-FG02-09R46554.
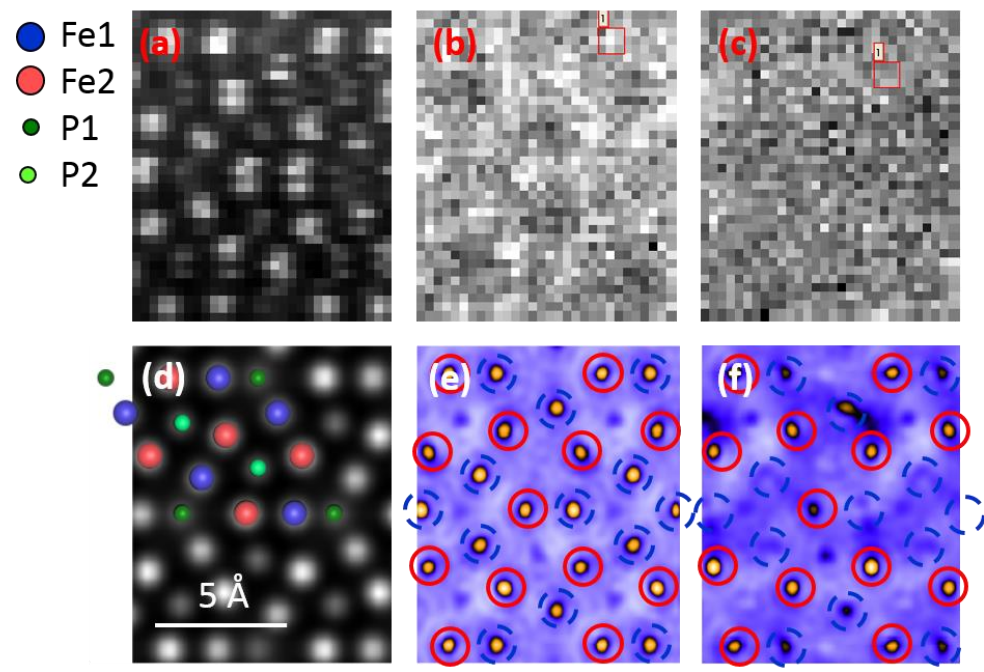

Figure 1. ADF image (a) and simultaneously acquired Fe L-shell and Mn L-shell spectrum images, (b) and (c) respectively, of $\mathrm{Mn}_{0.43} \mathrm{Fe}_{1.57} \mathrm{P}_{0.73} \mathrm{Si}_{0.27}$ (d) Simulated ADF image with structure inset. Recovered EELS scattering potentials for Fe L-shell and Mn L-shell ionization are shown in (e) and (f) respectively.
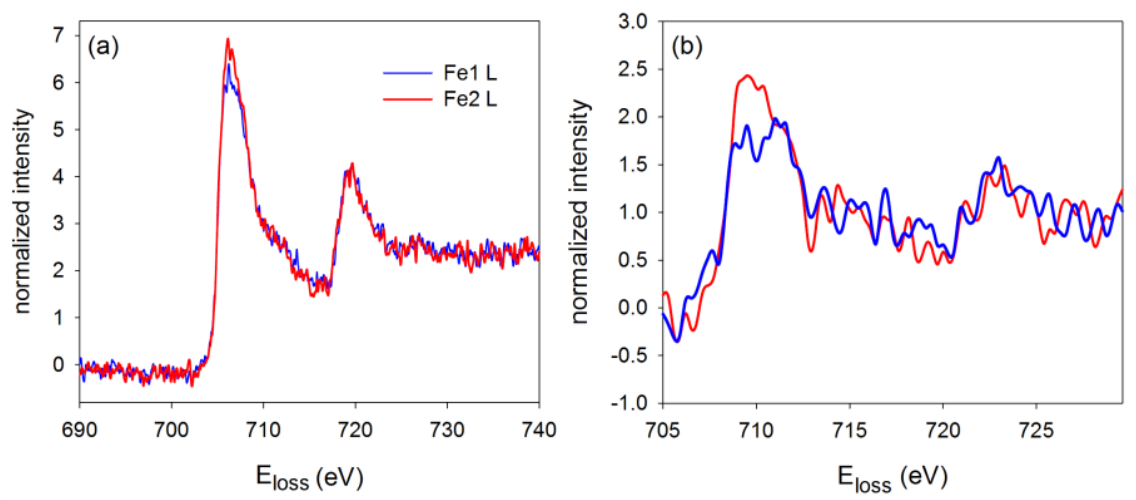

Figure 2. Fe1 L-shell (blue lines) and Fe2 L-shell (red lines) obtained from the raw data (a) and the inversion process (b). 